\title{
The Olympic Medals Ranks, lexicographic ordering and numerical infinities
}

\author{
Yaroslav D. Sergeyev ${ }^{1-3}$ \\ ${ }^{1}$ University of Calabria, Rende (CS), Italy \\ ${ }^{2}$ N.I. Lobatchevsky State University, Nizhni Novgorod, Russia \\ ${ }^{3}$ Institute of High Performance Computing and Networking \\ of the National Research Council of Italy, Rende (CS), Italy \\ yarodsi.dimes.unical.it
}

\begin{abstract}
Several ways used to rank countries with respect to medals won during Olympic Games are discussed. In particular, it is shown that the unofficial rank used by the Olympic Committee is the only rank that does not allow one to use a numerical counter for ranking - this rank uses the lexicographic ordering to rank countries: one gold medal is more precious than any number of silver medals and one silver medal is more precious than any number of bronze medals. How can we quantify what do these words, more precious, mean? Can we introduce a counter that for any possible number of medals would allow us to compute a numerical rank of a country using the number of gold, silver, and bronze medals in such a way that the higher resulting number would put the country in the higher position in the rank? Here we show that it is impossible to solve this problem using the positional numeral system with any finite base. Then we demonstrate that this problem can be easily solved by applying numerical computations with recently developed actual infinite numbers. These computations can be done on a new kind of a computer - the recently patented Infinity Computer. Its working software prototype is described briefly and examples of computations are given. It is shown that the new way of counting can be used in all situations where the lexicographic ordering is required.
\end{abstract}

Key Words: Olympic Games, medals ranks, lexicographic order, numeral systems, numerical infinities.

\section{Olympic medals ranks}

The International Olympic Committee (IOC) does not produce any official ranking of countries participating at the Olympic Games. However, the IOC publishes tables showing medals won by athletes representing each country participating at the Games. The convention used by the IOC to order the countries in this unofficial 
Table 1: The International Olympic Committee unofficial medal rank at Sochi 2014 (the first ten countries).

\begin{tabular}{|c|c|c|c|c|}
\hline Rank R1 & Country & Gold & Silver & Bronze \\
\hline 1 & Russian Federation & 13 & 11 & 9 \\
2 & Norway & 11 & 5 & 10 \\
3 & Canada & 10 & 10 & 5 \\
4 & United States & 9 & 7 & 12 \\
5 & Netherlands & 8 & 7 & 9 \\
6 & Germany & 8 & 6 & 5 \\
7 & Switzerland & 6 & 3 & 2 \\
8 & Belarus & 5 & 0 & 1 \\
9 & Austria & 4 & 8 & 5 \\
10 & France & 4 & 4 & 7 \\
\hline
\end{tabular}

rank is the following. First, countries are sorted by the number of gold medals won. If the number of gold medals is the same, the number of silver medals is taken into consideration, and then the number of bronze ones. If two countries have an equal number of gold, silver, and bronze medals, then equal ranking is given and the countries are listed alphabetically by their IOC country code (for instance, in the 2010 Winter Olympics held in Vancouver, China and Sweden have won 5 gold, 2 silver, and 4 bronze medals; both countries have the $7^{\text {th }}$ place in the rank but China is higher in the table). Table 1 shows countries sorted by using this rank at Sochi 2014 Olympic Games (the first ten countries). This rank will be called R1 hereinafter.

However, there exist several methods to rank countries (some of them are illustrated in Tables 2 and 3 showing best 10 countries for each rank, for more countries see, e.g., [3]). First, in many countries the total number of Olympic medals earned by athletes representing each country is very popular. This rank (called hereinafter R2) gives equal ratings to gold, silver, and bronze medals. So, if a country $A$ has won $g_{A}$ gold, $s_{A}$ silver, and $b_{A}$ bronze medals than its rank is just

$$
R 2(A)=g_{A}+s_{A}+b_{A} .
$$

Since this rank gives the same weight to gold, silver, and bronze medals, there are several proposals to improve this way of counting by introducing weights for medals. For instance, the Fibonacci weighted point system (this method is shown in Table 2 as R3) uses the following weights: gold costs 3 points, silver 2 points, and bronze 1 point, these weights are called 3:2:1 system. Thus, it follows

$$
R 3(A)=3 g_{A}+2 s_{A}+b_{A} .
$$

It can be seen from Table 2 that Norway and United States have the same rank R3 but in the rank Norway has a higher position because it has won more golds (the same situation holds for Switzerland and Sweden). In order to make gold medals more precious, the exponential weighted point system assigns 4 points to gold, 2 
Table 2: Medal ranks counting total number of won medals per country and weighted total sum (system 3:2:1).

\begin{tabular}{|c|cc|cc|}
\hline$N$ & Total medals (R2) & \multicolumn{2}{|c|}{ Weighted total medals (R3) } \\
\hline 1 & Russian Federation & 33 & Russian Federation & 70 \\
2 & United States & 28 & Canada & 55 \\
3 & Norway & 26 & Norway & 53 \\
4 & Canada & 25 & United States & 53 \\
5 & Netherlands & 24 & Netherlands & 47 \\
6 & Germany & 19 & Germany & 41 \\
7 & Austria & 17 & Austria & 33 \\
8 & France & 15 & France & 27 \\
9 & Sweden & 15 & Switzerland & 26 \\
10 & Switzerland & 11 & Sweden & 26 \\
\hline
\end{tabular}

Table 3: Medal ranks counting total medals per 10 million people and total medals per $\$ 100$ billion of the gross domestic product.

\begin{tabular}{|c|cr|cc|}
\hline$N$ & Total medals per $10^{7}$ people $(\mathrm{R} 4)$ & Total medals per \$100 billion of GDP (R5) \\
\hline 1 & Norway & 51.8 & Slovenia & 17.7 \\
2 & Slovenia & 38.9 & Latvia & 14.1 \\
3 & Austria & 20.1 & Belarus & 9.5 \\
4 & Latvia & 19.8 & Norway & 5.2 \\
5 & Sweden & 15.8 & Austria & 4.3 \\
6 & Netherlands & 14.3 & Czech Republic & 4.1 \\
7 & Switzerland & 13.8 & Netherlands & 3.1 \\
8 & Finland & 9.2 & Sweden & 2.9 \\
9 & Czech Republic & 7.6 & Finland & 2.0 \\
10 & Canada & 7.2 & Switzerland & 1.7 \\
\hline
\end{tabular}

points to silver, and 1 point to bronze $-4: 2: 1$ system. The variation employed by the British press during the Olympic Games in London in 1908 used the weights 5:3:1. There exist also systems 5:3:2, 6:2:1, 10:5:1, etc.

Ranks using completely different ideas should be also mentioned. For instante, it is possible to count all the medals won (weighted or not) counting separately the medals for each individual athlete in team sports. Another idea consists of the usage of an improvement rank that is based on percentage improvement reached by countries with respect to the previous Games results. There exist ranks built in comparison to expectations predictions. Among them, there can be mentioned predictions using previous results (during the Games or other competitions) and predictions using economics, population, and a range of other.

Another interesting proposal is to calculate the rank dividing the number of medals by the population of the country. The column R4 in Table 3 shows total medals won by a country divided by 10 million people. While criteria R1 - R3 give similar results, criterion R4 puts different countries, mainly having relatively small populations, on the top of the rank. In fact, Norway, that has won 26 medals 
and has a population of approximately 5 million people is the best in this rank. In general, the countries that top the list have also small populations in comparison, for instante, with United States and Russian Federation. The number of medals per $\$ 100$ billion of the gross domestic product (GDP) of the country (this rank is called R5 in Table 2) also favors smaller countries.

In this paper, we do not discuss advantages and disadvantages of various ranks. We consider a purely mathematical problem regarding the following difference that distinguishes the unofficial International Olympic Committee rank R1 and the other ranks R2 - R5. In fact, while ranks R2 - R5 produce numerical coefficients for each country that allow one to order the countries, rank R1 does not produce any resulting number that can be used for this purpose. This rank uses the so called lexicographic ordering called so because it is used in dictionaries to order words: first words are ordered with respect to the first symbol in the word, then with respect to the second one, and so on. In working with the rank R1 we have words that consists of three symbols $g_{A}, s_{A}, b_{A}$ and, therefore, their length $w=3$.

In this paper, we show that, as it happens for ranks R2 - R5, it is possible to propose a procedure for computing rank R1 numerically for each country and for any number of medals. Moreover, it is shown that the introduced way of computation can be generalized from words consisting of three symbols to words having a general finite length $w$ and used in situations that require the lexicographic ordering.

\section{How to compute the rank R1 for any number of medals?}

Evidently, in the rank R1 gold medals are more precious than silver ones that in their turn are better than the bronze ones. An interesting issue in this way of counting consists of the following fact. Let us consider Belarus and Austria that occupy the $8^{\text {th }}$ and $9^{\text {th }}$ positions, respectively. Belarus has 5 gold medals and Austria only 4. In spite of the fact that Austria has 8 silver medals and Belarus none of them, this fact is not taken into consideration. Austria could have any number of silver medals but one gold medal of Belarus will be more important than all these silver medals.

Can we quantify what do these words, more important, mean? Can we introduce in a way a counter that would allow us to compute a numerical rank of a country using the number of gold, silver, and bronze medals in such a way that the higher resulting number would put the country in the higher position in the rank? Moreover, we wish to construct a numerical counter that would work for any number of medals. This counter should work also in situations when the number of medals that can be won is not known a priori.

More formally, we would like to introduce a number $n\left(g_{A}, s_{A}, b_{A}\right)$ where $g_{A}$ is the number of gold medals, $s_{A}$ is the number of silver medals, and $b_{A}$ is the number of the bronze ones won by a country $A$. This number should be calculated 
in such a way that for countries $A$ and $B$ it follows that

$$
n\left(g_{A}, s_{A}, b_{A}\right)>n\left(g_{B}, s_{B}, b_{B}\right), \text { if }\left\{\begin{array}{l}
g_{A}>g_{B}, \\
g_{A}=g_{B}, s_{A}>s_{B}, \\
g_{A}=g_{B}, s_{A}=s_{B}, b_{A}>b_{0} .
\end{array}\right.
$$

In addition, $n\left(g_{A}, s_{A}, b_{A}\right)$ should be introduced under condition that the number $K>\max \left\{g_{A}, s_{A}, b_{A}\right\}$ being an upper bound for the number of medals of each type that can be won by each country is unknown.

In order to calculate $n\left(g_{A}, s_{A}, b_{A}\right)$, let us try to give weights to $g_{A}, s_{A}$, and $b_{A}$ as it is done in the positional numeral system with a base $\beta$ :

$$
n\left(g_{A}, s_{A}, b_{A}\right)=g_{A} \beta^{2}+s_{A} \beta^{1}+b_{A} \beta^{0}=g_{A} s_{A} b_{A}
$$

For instance, in the decimal positional numeral system with $\beta=10$ the record

$$
n\left(g_{A}, s_{A}, b_{A}\right)=g_{A} 10^{2}+s_{A} 10^{1}+b_{A} 10^{0}=g_{A} s_{A} b_{A}
$$

gives us the rank of the country $A$. However, it can be seen immediately that this way of reasoning does not solve our problem, since it does not satisfy condition (1). In fact, if a country will have more than 11 silver medals, then formula (3) implies that these medals are more important than one gold. For instance, the data

$$
g_{A}=2, s_{A}=0, b_{A}=0, \quad g_{B}=1, s_{B}=11, b_{B}=0 .
$$

give us

$$
\begin{aligned}
& n\left(g_{A}, s_{A}, b_{A}\right)=2 \cdot 10^{2}+0 \cdot 10^{1}+0 \cdot 10^{0}=200< \\
& n\left(g_{B}, s_{B}, b_{B}\right)=1 \cdot 10^{2}+11 \cdot 10^{1}+0 \cdot 10^{0}=210,
\end{aligned}
$$

i.e., condition (1) is not satisfied.

Remind that we wish to construct a numerical counter that would work for any number of medals, in other words, we suppose that countries can win any number of medals and this number is unknown for us. Then it is easy to see that situations can occur where the positional system will not satisfy (1) not only for the base $\beta=10$ but also for any finite $\beta$. This can happen if one of the countries will have more than $\beta$ silver (or bronze) medals.

Thus, the contribution of 1 gold medal in the computation of $n\left(g_{A}, s_{A}, b_{A}\right)$ should be larger than the contribution of any number, $s_{A}$, of silver medals, i.e., it should be infinitely larger. Analogously, the contribution of 1 silver medal should be infinitely larger than the contribution of any finite number of bronze medals.

Unfortunately, it is difficult to make numerical computations with infinity (symbolic ones can be done using non-standard analysis approach, see [10]) since in the traditional calculus $\infty$ absorbs any finite quantity and we have, for instance,

$$
\infty+1=\infty, \quad \infty+2=\infty .
$$




\section{A numerical calculator of the rank R1 involving infinities}

In order to construct a numerical calculator of medal rank involving infinite numbers, let us remind the difference between numbers and numerals: a numeral is a symbol or group of symbols that represents a number. The difference between them is the same as the difference between words and the things they refer to. A number is a concept that a numeral expresses. The same number can be represented by different numerals. For example, the symbols '7', 'seven', and 'VII' are different numerals, but they all represent the same number.

Different numeral systems can represent different numbers. For instance, Roman numeral system is not able to represent zero and negative numbers. There exist even weaker numeral systems. Recently (see [4]) a study on a numeral system of a tribe living in Amazonia - Pirahã - has been published. These people use a very simple numeral system for counting: one, two, many. For Pirahã, all quantities larger than 2 are just 'many' and such operations as $2+2$ and $2+1$ give the same result, i.e., 'many'. Using their weak numeral system Pirahã are not able to see, for instance, numbers $3,4,5$, and 6, to execute arithmetical operations with them, and, in general, to say anything about these numbers because in their language there are neither words nor concepts for that. It is important to emphasize that the records $2+1=$ 'many' and $2+2=$ 'many' are not wrong. They are correct in their language and if one is satisfied with the accuracy of the answer 'many', it can be used (and is used by Pirahã) in practice. Note that the result of Pirahã is not wrong, it is just inaccurate. Analogously, the answer 'many' to the question 'How many trees are there in a park?' is correct, but its precision is low.

Thus, if one needs a more precise result than 'many', it is necessary to introduce a more powerful numeral system allowing one to express the required answer in a more accurate way. By using numeral systems where additional numerals for expressing numbers 'three' and 'four' have been introduced, we can notice that within 'many' there are several objects and numbers 3 and 4 are among these unknown to Pirahã objects.

Our great attention to the numeral system of Pirahã is due to the following fact: their numeral 'many' gives them such results as

$$
\text { ‘many' }+1=\text { 'many', } \quad \text { 'many' }+2=\text { 'many', }
$$

that are very familiar to us, see (5). This comparison shows that we behave ourselves in front of infinity in the same way in which Pirahã behave themselves in front of quantities larger than 2 . Thus, our difficulty in working with infinity is not connected to the nature of infinity itself but is just a result of inadequate numeral systems that we use to work with infinity.

In order to avoid such situations as (5) and (6), a new numeral system has been proposed recently in [11, 13, 19, 23]. It is based on an infinite unit of measure expressed by the numeral (1) called grossone. A number of powerful theoretical and applied results have been obtained by several authors using the new methodology. For instance, the new approach has been compared with the panorama of 


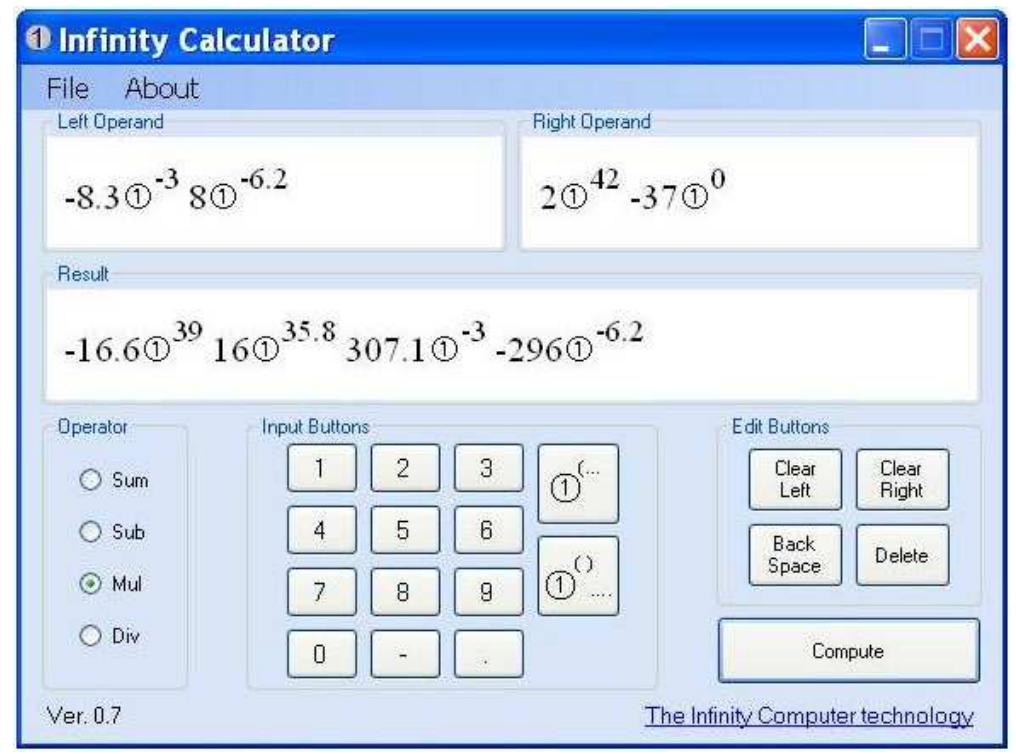

Figure 1: Operation of multiplication executed at the Infinity Calculator. The left operand has two infinitesimal parts, the right operand has an infinite part and a finite one; the result has two infinite and two infinitesimal parts.

ideas dealing with infinity and infinitesimals in [6-8, 25]. Then, the new methodology has been successfully applied for studying hyperbolic geometry (see [9]), percolation (see [5, 27]), fractals (see [12, 14, 22, 27]), numerical differentiation and optimization (see [1, 15, 20, 29]), infinite series and the Riemann zeta function (see [16, 21, 28]), the first Hilbert problem and Turing machines (see [18, 25, 26]), cellular automata (see [2]). The usage of numerical infinitesimals opens possibilities for creating new numerical methods having an accuracy that is superior to existing algorithms working only with finite numbers (see, e.g., algorithms for solving ordinary differential equations in [24]).

In particular, the Infinity Computer executing numerical computations with infinite and infinitesimal numbers has been patented (see [17]) and its software prototype has been constructed. This computer can be used to calculate the medal rank $n\left(g_{A}, s_{A}, b_{A}\right)$ satisfying condition (1) because it works with numbers expressed in the new positional numeral system with the infinite base (1). A number $C$ is subdivided into groups corresponding to powers of (1):

$$
C=c_{p_{m}}{ }^{1^{p_{m}}}+\ldots+c_{p_{1}}\left(^{1^{p_{1}}}+c_{p_{0}} 1^{p_{0}}+c_{p_{-1}}{ }^{1^{p_{-1}}}+\ldots+c_{p_{-k}}{ }^{1^{p_{-k}}} .\right.
$$

Then, the record

$$
C=c_{p_{m}}{ }^{1^{p_{m}}} \ldots c_{p_{1}} 1^{p_{1}} c_{p_{0}} 1^{p_{0}} c_{p_{-1}} 1^{p_{-1}} \ldots c_{p_{-k}}{ }^{1^{p_{-k}}}
$$

represents the number $C$. Numerals $c_{i} \neq 0$, they can be positive or negative and belong to a traditional numeral system and are called grossdigits. They show how 
many corresponding units $\left(1^{p_{i}}\right.$ should be added or subtracted in order to form the number $C$. Obviously, since all $c_{i}$ are finite, it follows

$$
\text { (1) }>c_{i} \text {. }
$$

Numbers $p_{i}$ in (8) are called grosspowers. They are sorted in the decreasing order

$$
p_{m}>p_{m-1}>\ldots>p_{1}>p_{0}>p_{-1}>\ldots p_{-(k-1)}>p_{-k}
$$

with $p_{0}=0$ and, in general, can be finite, infinite, and infinitesimal. Hereinafter we consider only finite values of $p_{i}$. Under this assumption, infinite numbers are expressed by numerals having at least one $p_{i}>0$. They can have several infinite parts, a finite part, and several infinitesimal ones. Finite numbers are represented by numerals having only one grosspower $p_{0}=0$. In this case $C=c_{0} \mathbb{1}^{0}=c_{0}$, where $c_{0}$ is a conventional finite number expressed in a traditional finite numeral system. Infinitesimals are represented by numerals $C$ having only negative grosspowers. The simplest infinitesimal is $\left(1^{-1}=\frac{1}{1}\right.$ being the inverse element with respect to multiplication for (1):

$$
\frac{1}{(1)} \cdot(1)=(1) \cdot \frac{1}{(1)}=1 \text {. }
$$

Note that all infinitesimals are not equal to zero. In particular, $\frac{1}{1}>0$ because it is a result of division of two positive numbers. Fig. 1 1 shows the Infinity Calculator built using the Infinity Computer technology.

It becomes very easy to calculate $n\left(g_{A}, s_{A}, b_{A}\right)$ using records (7), (8), i.e., putting (1) instead of a finite base $\beta$ in (2). Then the number

$$
n\left(g_{A}, s_{A}, b_{A}\right)=g_{A} \mathbb{1}^{2}+s_{A}{ }^{1}{ }^{1}+b_{A}{ }^{1}{ }^{0}=g_{A}{ }^{(1} s_{A}{ }^{1}{ }^{1} b_{A}{ }^{1}{ }^{0}
$$

gives us the rank of the country satisfying condition (11). Let us consider as an example the data (4). Since (1) is larger than any finite number (see (9)), it follows from (11) that

$$
\begin{gathered}
n\left(g_{A}, s_{A}, b_{A}\right)=2 \cdot\left(1^{2}+0 \cdot(1)^{1}+0 \cdot\left(1^{0}=2(1)^{2}>\right.\right. \\
n\left(g_{B}, s_{B}, b_{B}\right)=1 \cdot(1)^{2}+11 \cdot(1)^{1}+0 \cdot(1)^{0}=1(1)^{2} 11(1)^{1}
\end{gathered}
$$

since

$$
2\left(1^{2}-11^{2} 111^{1}=11^{2}-11(1)^{1}=1(1)-11\right)>0 .
$$

Thus, we can easily calculate the rank R1 for the data from Table 1 as follows

$$
\begin{aligned}
& 13(1)^{2} 11(1)^{1} 9(1)^{0}>111^{2} 5(1)^{1} 10(1)^{0}>101^{2} 10(1)^{1} 5(1)^{0}>91^{2} 7(1)^{1} 12(1)^{0}> \\
& 8\left(1^{2} 7(1)^{1} 9(1)^{0}>8\left(1^{2} 6(1)^{1} 5(1)^{0}>6\left(1 ^ { 2 } 3 ( 1 ) ^ { 1 } 2 \left(1^{0}>5(1)^{2} 0(1)^{1} 11^{0}>\right.\right.\right.\right. \\
& 4(1)^{2} 8(1)^{1} 51^{0}>4(1)^{2} 4(1)^{1} 7(1)^{0} \text {. }
\end{aligned}
$$

We can conclude so that the introduced calculator can be used for computing the unofficial International Olympic Committee rank R1 numerically. Clearly, it can also be applied in all situations that require the lexicographic ordering not only for words with three characters as it happens for the rank R1 but for words having any finite number of characters, as well. 


\section{References}

[1] S. De Cosmis and R. De Leone. The use of grossone in mathematical programming and operations research. Applied Mathematics and Computation, 218(16):8029-8038, 2012.

[2] L. D'Alotto. Cellular automata using infinite computations. Applied Mathematics and Computation, 218(16):8077-8082, 2012.

[3] R. Florida. Did Russia really win the Sochi medal count? The Atlantic, February 23, 2014.

[4] P. Gordon. Numerical cognition without words: Evidence from Amazonia. Science, 306(15 October):496-499, 2004.

[5] D.I. Iudin, Ya.D. Sergeyev, and M. Hayakawa. Interpretation of percolation in terms of infinity computations. Applied Mathematics and Computation, 218(16):8099-8111, 2012.

[6] G. Lolli. Infinitesimals and infinites in the history of mathematics: A brief survey. Applied Mathematics and Computation, 218(16):7979-7988, 2012.

[7] G. Lolli. Metamathematical investigations on the theory of grossone. Applied Mathematics and Computation, to appear.

[8] M. Margenstern. Using grossone to count the number of elements of infinite sets and the connection with bijections. p-Adic Numbers, Ultrametric Analysis and Applications, 3(3):196-204, 2011.

[9] M. Margenstern. An application of grossone to the study of a family of tilings of the hyperbolic plane. Applied Mathematics and Computation, 218(16):8005-8018, 2012.

[10] A. Robinson. Non-standard Analysis. Princeton Univ. Press, Princeton, 1996.

[11] Ya.D. Sergeyev. Arithmetic of Infinity. Edizioni Orizzonti Meridionali, CS, 2003.

[12] Ya.D. Sergeyev. Blinking fractals and their quantitative analysis using infinite and infinitesimal numbers. Chaos, Solitons \& Fractals, 33(1):50-75, 2007.

[13] Ya.D. Sergeyev. A new applied approach for executing computations with infinite and infinitesimal quantities. Informatica, 19(4):567-596, 2008.

[14] Ya.D. Sergeyev. Evaluating the exact infinitesimal values of area of Sierpinski's carpet and volume of Menger's sponge. Chaos, Solitons \& Fractals, 42(5):3042-3046, 2009. 
[15] Ya.D. Sergeyev. Numerical computations and mathematical modelling with infinite and infinitesimal numbers. Journal of Applied Mathematics and Computing, 29:177-195, 2009.

[16] Ya.D. Sergeyev. Numerical point of view on Calculus for functions assuming finite, infinite, and infinitesimal values over finite, infinite, and infinitesimal domains. Nonlinear Analysis Series A: Theory, Methods \& Applications, 71(12):e1688-e1707, 2009.

[17] Ya.D. Sergeyev. Computer system for storing infinite, infinitesimal, and finite quantities and executing arithmetical operations with them. USA patent 7,860,914, 2010.

[18] Ya.D. Sergeyev. Counting systems and the First Hilbert problem. Nonlinear Analysis Series A: Theory, Methods \& Applications, 72(3-4):1701-1708, 2010.

[19] Ya.D. Sergeyev. Lagrange Lecture: Methodology of numerical computations with infinities and infinitesimals. Rendiconti del Seminario Matematico dell'Università e del Politecnico di Torino, 68(2):95-113, 2010.

[20] Ya.D. Sergeyev. Higher order numerical differentiation on the infinity computer. Optimization Letters, 5(4):575-585, 2011.

[21] Ya.D. Sergeyev. On accuracy of mathematical languages used to deal with the Riemann zeta function and the Dirichlet eta function. p-Adic Numbers, Ultrametric Analysis and Applications, 3(2):129-148, 2011.

[22] Ya.D. Sergeyev. Using blinking fractals for mathematical modelling of processes of growth in biological systems. Informatica, 22(4):559-576, 2011.

[23] Ya.D. Sergeyev. Numerical computations with infinite and infinitesimal numbers: Theory and applications. In Sorokin A. and Pardalos P.M., editors, Dynamics of Information Systems: Algorithmic Approaches, pages 1-66. Springer, New York, 2013.

[24] Ya.D. Sergeyev. Solving ordinary differential equations by working with infinitesimals numerically on the infinity computer. Applied Mathematics and Computation, 219(22):10668-10681, 2013.

[25] Ya.D. Sergeyev and A. Garro. Observability of Turing machines: A refinement of the theory of computation. Informatica, 21(3):425-454, 2010.

[26] Ya.D. Sergeyev and A. Garro. Single-tape and multi-tape Turing machines through the lens of the Grossone methodology. Journal of Supercomputing, 65(2):645-663, 2013. 
[27] M.C. Vita, S. De Bartolo, C. Fallico, and M. Veltri. Usage of infinitesimals in the Menger's Sponge model of porosity. Applied Mathematics and Computation, 218(16):8187-8196, 2012.

[28] A.A. Zhigljavsky. Computing sums of conditionally convergent and divergent series using the concept of grossone. Applied Mathematics and Computation, 218(16):8064-8076, 2012.

[29] A. Žilinskas. On strong homogeneity of two global optimization algorithms based on statistical models of multimodal objective functions. Applied Mathematics and Computation, 218(16):8131-8136, 2012. 